\title{
Low-dose computed tomography of urolithiasis in obese patients: a feasibility study to evaluate image reconstruction algorithms
}

This article was published in the following Dove Press journal:

Diabetes, Metabolic Syndrome and Obesity: Targets and Therapy

\section{De-Hua Chang ${ }^{l, *}$ \\ Karin Slebocki ${ }^{2, *}$ \\ Ekaterina Khristenko' \\ Jan Herden ${ }^{3}$ \\ Johannes Salem ${ }^{3}$ \\ Nils Große Hokamp ${ }^{2}$ \\ Kamal Mammadov ${ }^{2}$ \\ Martin Hellmich ${ }^{4}$ \\ Christoph Kabbasch ${ }^{2}$ \\ 'Department of Diagnostic and Interventional Radiology, University Medical Center Heidelberg, Heidelberg, Germany; ${ }^{2}$ Department of Diagnostic and Interventional Radiology, University Hospital of Cologne, Cologne, Germany; ${ }^{3}$ Department of Urology, University Hospital of Cologne, Cologne, Germany; ${ }^{4}$ Institute of Medical Statistics and Computational Biology (IMSB), Medical Faculty, University of Cologne, Cologne, Germany}

*These authors contributed equally to this work
Correspondence: De-Hua Chang Im Neuenheimer Feld I 10, Heidelberg 69120, Germany

Email de-hua.chang@med.uni-heidelberg.de
Purpose: Retrospective evaluation and comparison of image quality generated by low-dose computed tomography (LDCT) from obese patients with urolithiasis using alternative reconstruction algorithms.

Materials and methods: Twenty-five obese patients (body mass index [BMI] $>25 \mathrm{~kg} / \mathrm{m}^{2}$ ) underwent LDCT scans for suspected urolithiasis. The scans were recompiled using filteredback projection (FBP), statistical iterative reconstruction (iDose) and iterative model-based reconstruction (IMR). Dose-length product (DLP) and patient details were obtained from the CT dose report and clinical charts, respectively. Objective image noise was assessed by measuring the SD of Hounsfield units (HUs) in defined locations. Additionally, subjective image evaluation was independently performed by two radiologists using a 3-point Likert scale. The inter-reviewer agreement of image quality was calculated.

Results: Ureteral concretions were observed in all CT scans, two of which revealed bilateral stones. The assessed patients' mean BMI was $29.29 \pm 3.74 \mathrm{~kg} / \mathrm{m}^{2}$, and the DLP of the CT scans was $100.04 \pm 10.00 \mathrm{mGy}^{*} \mathrm{~cm}$. All scans were rated diagnostic with the iDose and iterative model-based reconstructions, whereas $41 \%$ of the scans performed with FBP reconstruction were nondiagnostic. With respect to image quality, IMR was superior to iDose and FBP, both in the objective $(P<0.001)$ and overall subjective $(P \leq 0.008)$ evaluation of the respective data sets. The inter-reviewer agreement for overall image quality was "almost perfect" for IMR, "substantial" for iDose and "moderate" for FBP ( $\kappa$ values of $1.0,0.6$ and 0.46 , respectively).

Conclusion: Using iterative image reconstruction algorithms, LDCT of urolithiasis is feasible in overweight patients with a BMI between 25 and $35 \mathrm{~kg} / \mathrm{m}^{2}$. Due to higher image quality, IMR is the preferred algorithm for scan reconstruction as it may help to avoid repeated examinations due to initial nondiagnostic scans.

Keywords: low-dose computed tomography, model-based iterative reconstruction, statistical iterative, urolithiasis, obesity

\section{Background}

Urolithiasis is a common disease with increasing incidence, prevalence and reoccurrence in industrialized countries. The rise in renal stone disease is particularly evident in younger patients and is primarily due to factors such as overconsumption of food, sedentary lifestyle, increased fructose intake and reduced physical activity. ${ }^{1}$ Statistics reveal that up to $12 \%$ of the population will contract a urinary stone during their lifetime, with recurrence rates amounting around $50 \%{ }^{2}$ Fifty-five 
percent of patients with recurrent stones have a family history of urolithiasis. ${ }^{3}$ Consequently, these patients have a three-fold risk of developing stones. ${ }^{4}$ Epidemiological studies have revealed that the incidence of stone risk may rise with increasing body mass index (BMI). ${ }^{1}$

With a sensitivity of $95-96 \%$ and a specificity of 97-100\%, an unenhanced computed tomography (CT) scan of the abdomen and pelvis is considered a state-of-the-art method to confirm or eliminate suspected urolithiasis in symptomatic patients. $^{5-7}$ As diagnosis by CT has become more widespread, radiation dose in patients has become a major concern. ${ }^{8-10}$ Consequently, attention has recently been directed to using low-dose CT protocols in order to reduce the effective radiation dose administered to the patient. ${ }^{8}$ These low-dose scans have been increasingly implemented in routine clinical investigations to diagnose urinary calculi. Moreover, ultra-low-dose CT protocols (ULDCT) with effective doses $<1$ millisievert ( $\mathrm{mSv}$ ) have been evaluated in several studies and have shown promising results. ${ }^{9,10}$ For individuals with high BMI scores, low-dose CT imaging is often restricted because of excessive image noise. Even when using high radiation doses, accurate diagnosis of urolithiasis is often difficult in overweight and obese patients. $^{11}$

In order to address the increasing concern of cancer risk through multiple CT procedures, combined with the difficulties associated with scanning obese patients using standard filtered-back projection (FBP), several CT image reconstruction algorithms have been developed and implemented across a range of clinical scenarios. ${ }^{1-14}$ These include statistical iterative reconstruction algorithms and the more recent model-based iterative reconstruction algorithms. Statistical iterative reconstruction algorithms perform multiple repetitions to reduce image noise and improve the signal-to-noise ratio of raw CT data. In contrast, model-based iterative reconstruction algorithms use the statistical model of noise and in addition the geometry of the acquisition process, including voxel volumes of the scanned object. By increasing the signal-to-noise ratio, both processes have led to significant improvements in image quality in normal-weight and obese patients at reduced radiation doses. ${ }^{11-14}$

The aim of this study was to assess an LDCT protocol for obese patients with renal stones. The quality of the CT image and subsequent diagnostic confidence was evaluated using three different reconstruction algorithms (FBP, iDose and iterative model-based reconstruction [IMR]).

\section{Materials and methods}

This retrospective study was conducted at a German tertiary care hospital and over a period of 11 months. We assessed data from consecutive patients, who had received CT examination due to suspected symptomatic ureterolithiasis. All patients who were older than 18 years and had a BMI $>25 \mathrm{~kg} / \mathrm{m}^{2}$ were enrolled onto the study. Patient characteristics, clinical course (conservative vs invasive therapy) and diagnosis at the time of discharge were obtained from patient records. The dose-length product (DLP) was extracted from the $\mathrm{CT}$ report. In addition, effective radiation doses were calculated as $\mathrm{mSv}$ from the recorded DLP with a conversion factor of $0.0151 .^{15}$

This study was approved by the institutional review board of the medical faculty, University of Cologne. As the study was retrospective and observational, patient written informed consent was waived by the board.

\section{CT technique}

All examinations were performed using a 256-row computed tomography scanner (iCT, Philips Healthcare, Cleveland, OH, USA) with $128 \times 0.625 \mathrm{~mm}$ section collimation and a pitch factor of 0.977 . The scans were performed at a tube voltage of $100 \mathrm{kV}$ and a fixed tube current of $50 \mathrm{mAs}$. The scan range included the sub-phrenic space to the symphysis.

The acquired raw data was recompiled using a filtered back reconstruction algorithm (FBP) (Philips Healthcare, Cleveland, OH, USA), a statistical iterative reconstruction algorithm (iDose, Philips Healthcare) with iteration level 4, and a model-based iterative reconstruction algorithm (IMR, Philips Healthcare) with soft tissue level 2.

\section{Image analysis}

All image reconstructions were anonymized and independently evaluated by two radiologists trained in gastrointestinal imaging with diagnostic experience of 8 and 3 years, respectively. Both radiologists were masked, both with regard to patient outcome and their companion's image interpretation. Images were evaluated in a random order, and image quality was rated on a 3-point scale as follows:

1. Overall image quality: $1=$ nondiagnostic, interpretation not possible; $2=$ severely reduced image quality, restricted interpretation; $3=$ no/slightly reduced image quality, interpretation unequivocal 
2. Delineation of the ureter: $1=$ nondiagnostic, delineation of the ureter not possible; 2 = delineation of the ureter questionable; $3=$ delineation of the ureter possible

3. Image noise: $1=$ severe, interpretation not possible; 2 = moderate, interpretation limited; $3=$ minimal $/$ little, interpretation clear

4. Sharpness/outline of concretion: $1=$ severely blurred; 2 = slightly blurred; $3=$ sharply defined

In the IMR data sets, number (n), location (side: left-right, ureter: proximal-middle-distal), and size $(<5 \mathrm{~mm}$ or $\geq 5 \mathrm{~mm}$ ) were independently assessed by both reviewers.

Defined as SD of Hounsfield units (HUs), image noise was used as a parameter for objective image quality. For all scans, circular regions of interest (ROIs) with an area of $100 \mathrm{~mm}^{2}$ were drawn on the psoas muscle and the gluteal subcutaneous fat at the level of the fifth lumbar vertebra/first sacral vertebra by a 4 th year radiology resident.

\section{Statistical analyses}

Quantitative and qualitative variables were summarized by mean \pm SD and by count (percentage), respectively. Differences in distribution between the reconstruction algorithms were evaluated using the Wilcoxon signed-rank or Friedman test. Difference (or agreement) between reviewers was evaluated by McNemar's test or the kappa statistic. $P$-values $<0.05$ were assumed indicative of statistical significance. Inter-reviewer agreement was interpreted according to the following scheme: "poor", $\kappa$ value $\leq 0.19$; "fair", $\kappa$ value $0.20-0.39$; "moderate", $\kappa$ value $0.40-0.59$; "substantial", $\kappa$ value $0.60-0.79$ and "almost perfect", $\kappa$ value $0.80-1.00$. All calculations were performed with SPSS Statistics (IBM Corporation, Armonk, NY, USA).

\section{Results}

Twenty-five consecutive patients with suspected urolithiasis were enrolled in this study. The patient BMI was 29.29 $\pm 3.74 \mathrm{~kg} / \mathrm{m}^{2}$ (range: $25.2-36.0 \mathrm{~kg} / \mathrm{m}^{2}$ ). Patient data and imaging analysis, including inter-reviewer agreement $(\kappa)$, are summarized in Table 1. In each scan, ureteral concretions were consistently detected by both radiologists, and two revealed bilateral stones. Subsequently, urolithiasis was the final clinical diagnosis at the time of discharge for all patients. For 17 cases, conservative treatment with analgesic medication and administration of intravenous
Table I Patient distribution and imaging data

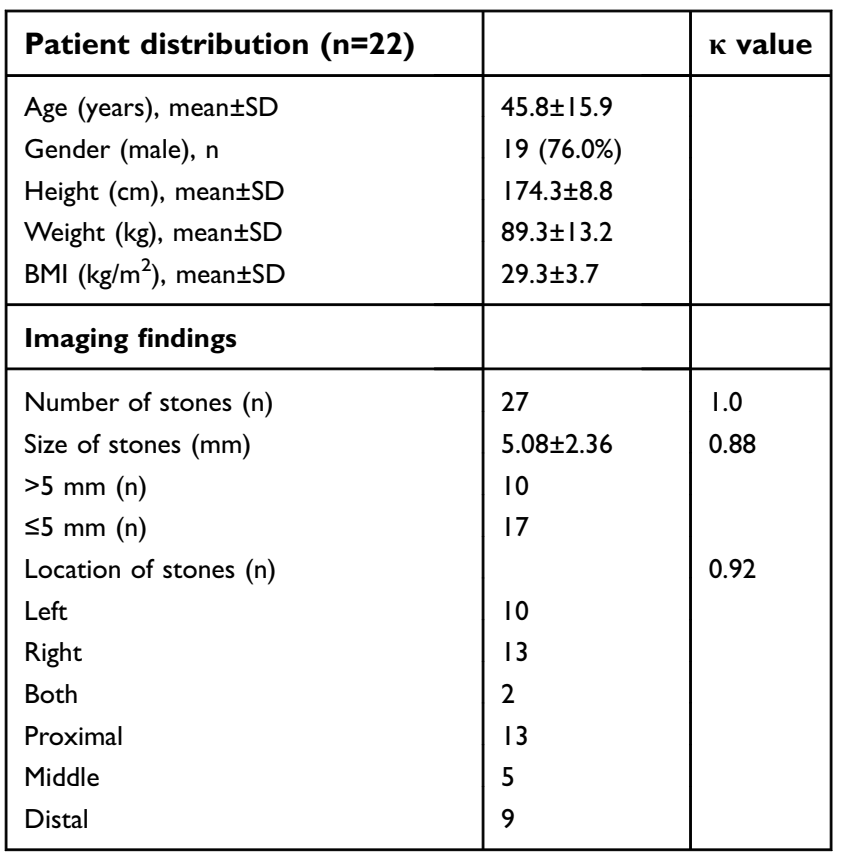

Note: For simplicity, only the imaging results from reviewer I are given. Abbreviation: BMI, body mass index.

infusion led to a spontaneous recovery. Six patients underwent ureteroscopy. Furthermore, in 4 cases, double-J stents were inserted. One patient underwent percutaneous nephrolithotomy and another, laser lithotripsy.

The DLP and effective radiation doses of the CT scans were $100.04 \pm 10.00 \mathrm{mGy}^{*} \mathrm{~cm}$ and $1.51 \pm 0.15 \mathrm{mSv}$, respectively.

\section{Image analysis}

Table 2 shows the subjective image evaluation for each category (overall image quality, delineation of ureter, image noise and sharpness/outline of concretion) using the three different reconstruction algorithms. In summary, IMR was superior to iDose and FBP across all categories $(P \leq 0.008)$. Excluding delineation of the ureter, the interreviewer agreement was highest for IMR across all categories (Table 2).

All scans were rated as diagnostic in the iDose and IMR reconstructed images, whereas 9 out of 22 scans (41\%) belonging to the FBP images were rated as nondiagnostic.

The objective image noise was lowest in the IMR group (both in muscle and fat) followed by iDose and FBP (Table 3). In relative terms compared to IMR, image noise was 2.5- and 4.1-fold higher by iDose and FBP, respectively, in muscle and 2.2- and 3.9-fold in fat. 
Table 2 Image evaluation scores (mean $\pm S D$ ) and inter-reviewer agreement (к). Overall image quality: $\mathrm{I}=$ nondiagnostic, interpretation not possible; 2 = severely reduced image quality, restricted interpretation; 3 = no/slightly reduced image quality, interpretation unequivocal. Delineation of ureter: $\mathrm{I}=$ nondiagnostic, delineation of ureter not possible; 2 = delineation of ureter questionable; 3 = delineation of ureter possible. Image noise: $\mathrm{I}$ = severe, interpretation not possible; 2 = moderate, interpretation limited; $3=\mathrm{minimal} / \mathrm{little}$, interpretation clear. Sharpness/outline of concretion: I = severely blurred, 2 = slightly blurred, 3 = sharply defined

\begin{tabular}{|l|l|l|l|}
\hline & IMR & iDose & FBP \\
\hline Overall image quality & $3.0 \pm 0.2$ & $2.6 \pm 0.5$ & $1.6 \pm 0.6$ \\
$\kappa$ & $\mathrm{I}$ & 0.60 & 0.46 \\
Delineation of ureter & $2.8 \pm 0.5$ & $2.1 \pm 0.5$ & $\mathrm{I} .3 \pm 0.5$ \\
$\kappa$ & 0.52 & $0.6 \mathrm{I}$ & 0.52 \\
Image noise & $2.3 \pm 0.5$ & $1.4 \pm 0.5$ & $1.0 \pm 0.0$ \\
$\kappa$ & 0.44 & 0.27 & $\mathrm{n} / \mathrm{a}$ \\
Sharpness/outline of concretion & $2.1 \pm 0.6$ & $1.6 \pm 0.5$ & $\mathrm{I} .0 \pm 0.2$ \\
$\kappa$ & 0.48 & 0.26 & -0.04 \\
\hline
\end{tabular}

Note: For simplicity, only the imaging data from reviewer I are given.

Abbreviations: n/a, not available; FBP, filtered-back projection; IMR, iterative model-based reconstruction.

Table 3 Objective image noise (mean \pm SD)

\begin{tabular}{|l|l|l|l|}
\hline & FBP & iDose & IMR \\
\hline Muscle & $89.4 \pm 20.9$ & $55.1 \pm 10.5$ & $21.7 \pm 5.4$ \\
Fat & $78.2 \pm 23.1$ & $43.4 \pm 7.3$ & $19.6 \pm 3.5$ \\
\hline
\end{tabular}

Abbreviations: FBP, filtered-back projection; IMR, iterative modelbased reconstruction.

\section{Discussion}

In recent decades, there has been a steady increase in the incidence of urolithiasis in many industrialized countries. $^{16,17}$ In parallel, a consistent rise in the number of overweight and obese people is apparent and has now reached epidemic proportions. ${ }^{18,19}$ Perhaps even more alarming is a clear and rising trend in the incidence of young patients suffering from urolithiasis and that this observation can, in part, be explained by lifestyle changes, which include overconsumption of food and reduced physical activity. Indeed, there is mounting evidence that obesity or being overweight at a young age are closely associated with an increased likelihood of developing kidney stones. ${ }^{20,21}$

The rise in urolithiasis and their recurrence in overweight and obese patients is associated with the concurrent increase in the number of times a patient undergoes a CT protocol. Therefore, minimizing radiation exposure is imperative for all patients, but particularly relevant for younger patients where there is a longer lifetime risk associated with contracting cancer. $^{22,23}$ For patients that present with acute renal colic, low-dose computed tomography (LDCT) represents the current gold standard. ${ }^{23,24}$ In addition, studies have been performed to compare radiation dose and image quality via iterative reconstruction algorithms in standard-dose (SD), low-dose (LD), and ultralowdose (ULD) CT protocols to evaluate diagnostic performance of LDCT for urolithiasis. ${ }^{25,26}$

However, to our knowledge, none of these studies have focused exclusively on evaluating the image quality of LDCT scans of obese patients with suspected urolithiasis.

It should be noted that the terms "ultra-low-dose", "low-dose" and "standard-dose" are not clearly defined by the radiological societies and are used diversely in literature. Nevertheless, in most abdominal studies, effective doses $<1 \mathrm{mSv}, 1-4 \mathrm{mSv}$ and 4-8 $\mathrm{mSv}$ are considered ULD, LD and SD, respectively. ${ }^{25,26}$ Therefore, for a normal-weight individual, the estimated dose reduction for a ULDCT scan relative to a SD CT is approximately $75-85 \%$. These definitions, however, are not always applicable to CT scans performed on obese patients. Park et al indicated an average effective radiation dose of $10.3 \pm 2.42 \mathrm{mSv}$ was required for a standard abdominal CT on obese patients (BMI $25-35 \mathrm{~kg} / \mathrm{m}^{2}$ ). In our study, however, the mean effective radiation dose was $1.51 \pm 0.15 \mathrm{mSv}$, representing a reduction of approximately $85 \%$. This decrease was achieved by using a CT protocol with a lowered tube voltage of $100 \mathrm{kVp}$ and a fixed tube current-time product.

In addition to reducing patient exposure to radiation, our data stress the key importance of postprocessing CT images via iterative reconstruction algorithms to reach an accurate diagnosis. For obese individuals, CT imaging is often inferior due to excessive image noise and artifacts. Consistent with this well-known fact, we observed that without postprocessing of the images, $41 \%$ of the FBP data sets were subjectively rated as nondiagnostic (Figure 1).

Similar to our findings, other studies have demonstrated the superiority of model-based iterative reconstructions on image quality, ${ }^{25,27,28}$ particularly for ULD-CT scans. ${ }^{25,27}$ Hur et $\mathrm{al}^{25}$ compared radiation dose and image quality in normalweight patients with urolithiasis. All underwent three CT scans under reduced dose (RD), LD and ULD protocols. RD scans were reconstructed using FBP, whilst LD scans were reconstructed using a statistical iterative algorithm. ULD scans were reconstructed using both techniques and a model-based 

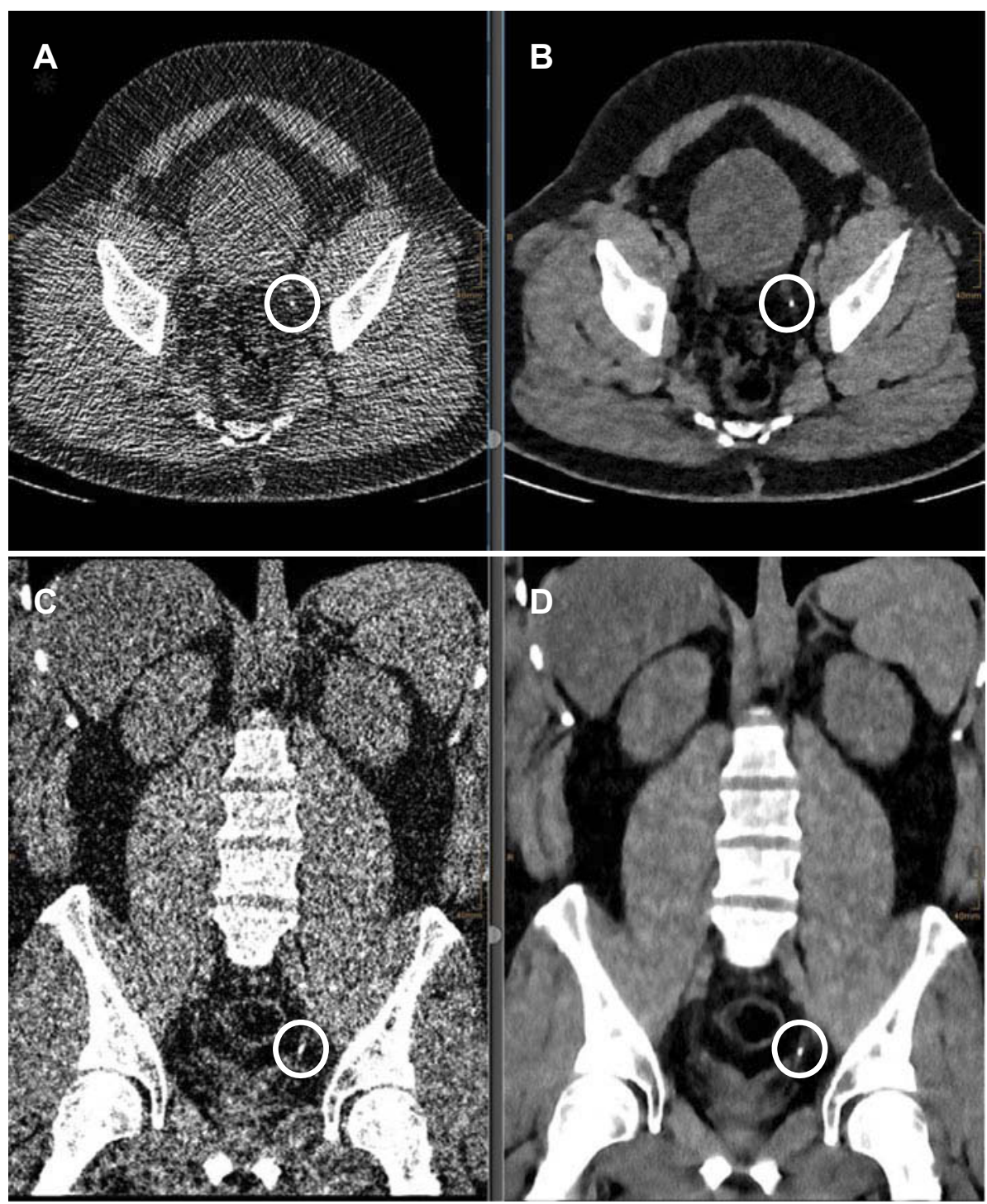

Figure I Unenhanced low-dose CT of the abdomen. A 30-year old, male patient with BMI of 36. Effective dose: I.62 mSv. (A, C) Severe image noise resulting in restricted interpretation on axial and coronal reformation with FBP). (B, D) Same patient with iterative model-based reconstruction (IMR). Concrement (I mm) encircled in the distal ureter on the left side, only clearly visible on IMR image set.

Abbreviations: CT, computed tomography; FBP, filtered-back projection; IMR, iterative model-based reconstruction.

iterative reconstruction. The model-based iterative reconstruction algorithm gave rise to lower subjective image quality compared to the $\mathrm{RD}$ and $\mathrm{LD}$ protocols but showed the least objective image noise. Overall, LD images show a $>84 \%$ concordance rate and $100 \%$ in ureter stones $>3 \mathrm{~mm}$. Interobserver agreement was substantial ( $\kappa$ value $=0.61$ ).

Botsikas et $\mathrm{al}^{28}$ showed that ureteral definition was significantly improved for model-based iterative reconstruction compared to adaptive statistical iterative reconstruction. As a better delineation of the ureter helps in localization of the stones, it is beneficial for the diagnosis and therefore a desirable effect of model-based iterative reconstruction. The mean contrast-to-noise ratio was also significantly improved with the model-based iterative reconstruction compared to the adaptive statistical iterative reconstruction.

Despite the positive results obtained in our study and the clear advantages of iterative reconstruction algorithms, there are two drawbacks associated with this approach. First, reconstruction time is the longest in IMR followed by iDose and FBP. According to the specifications of the manufacturer and the capacity of the user hardware, the reconstruction speeds are $33 \mathrm{~mm} / \mathrm{s}$ and $24 \mathrm{~mm} / \mathrm{s}$ for FBP and iDose, respectively. In a previous study, the reconstruction time was abrogated in 52 patients undergoing CT-guided lung interventions. ${ }^{13}$ The authors concluded that relative to iDose, FBP reduces reconstruction time by approximately $30 \%$. Khawaja et al 
demonstrated that IMR took $<5 \mathrm{~min}$ at an offline reconstruction facility, but was considerably shorter on the CT user interface (approximately $3 \mathrm{~min}$ for the majority of reference protocols). ${ }^{29}$ With respect to patient safety and workflow, the absolute reconstruction speed is tolerable for all reconstruction algorithms, since patients with suspected urolithiasis are not usually considered as emergency cases.

A second shortcoming is that IMR has a tendency to overevaluate the density and size of the stones. Botsikas et al showed that the maximal density measured from modelbased iterative reconstruction was significantly higher (754.4 $\pm 376.5 \mathrm{HU})$ than that measured from adaptive statistical iterative reconstruction $(559.4 \pm 352.4 \mathrm{HU})$. The size of the stones was overevaluated with model-based iterative reconstruction (mean diameter, $4.91 \pm 2.61 \mathrm{~mm}$ ) compared to adaptive statistical iterative reconstruction $(4.52 \pm 2.63 \mathrm{~mm}) .^{28}$ Depending on the type of reconstruction algorithm applied, we also observed that the sharpness/outline of the concretion was mostly rated as being slightly to severely blurred. Moreover, the inter-reviewer agreement in this category was only poor to moderate $(-0.04$ to 0.48$)$.

Despite these observations, an almost perfect inter-reviewer agreement was achieved with the measurement of stone size in the IMR data sets. This may be attributable to the fact that only a few stones $<3 \mathrm{~mm}$ in size were observed. Calcified stones $>5 \mathrm{~mm}$ are usually detected reliably by CT. Clinically, additional diagnostic or therapeutic procedures may not be required because in up to $98 \%$ of cases, stones $<5 \mathrm{~mm}$ usually pass spontaneously. ${ }^{30}$

Our study has a few limitations. First, a retrospective study with a small study population was conducted without a control group. Therefore, radiation exposure is only approximately comparable to other studies. Obviously, patient characteristics, scanning protocols and reconstruction techniques with different vendors may restrict the comparability of the results. Furthermore, a dose-finding study was not performed. Therefore, the extent to which radiation dose could be further reduced remains unclear and could be investigated in a potential follow-up study. Nevertheless, the fact that the image quality of all examinations was rated as being of diagnostic quality, indicating that even further dose reduction is reasonable and should be considered. Severely obese patients $\left(\mathrm{BMI}>35 \mathrm{~kg} / \mathrm{m}^{2}\right)$ were not well-represented in this study and, as such, adapted protocols may still be required for this patient group. Furthermore method inherent there might have been false-negative findings in non calcified stones. In some patients, excretion of ureteral stones was not obtained as a reference standard. In these cases, the final diagnosis of urolithiasis was made when symptoms spontaneously resolved an by exclusion of other underlying diseases.

\section{Conclusion}

Using iterative reconstruction algorithms, LDCT of urolithiasis is feasible in overweight patients with a BMI between 25 and $35 \mathrm{~kg} / \mathrm{m}^{2}$. With respect to image quality, IMR emerges to be the preferred algorithm and, as such, may alleviate the need for repeated examinations due to previously measured nondiagnostic scans.

\section{Disclosure}

Dr DH. Chang and Dr N. Große Hokamp report personal fees from Philips Healthcare outside the submitted work. The other authors report no conflicts of interest in this work.

\section{References}

1. Asplin JR. Obesity and urolithiasis. Adv Chronic Kidney Dis. 2009;16(1):11-20. doi:10.1053/j.ackd.2008.10.003

2. Sierakowski R, Finlayson B, Landes RR, Finlayson CD, Sierakowski $\mathrm{N}$. The frequency of urolithiasis in hospital discharge diagnoses in the United States. Invest Urol. 1978;15(6):438-441.

3. Ljunghall S, Danielson BG, Fellström B, Holmgren K, Johansson G, Wikström B. Family history of renal stones in recurrent stone patients. Br J Urol. 1985;57(4):370-374.

4. Curhan GC, Willett WC, Rimm EB, Stampfer MJ. Family history and risk of kidney stones. J Am Soc Nephrol. 1997;8(10):1568-1573.

5. Türk C, Petřík A, Sarica K, et al. EAU guidelines on interventional treatment for urolithiasis. Eur Urol. 2016;69(3):475-482. doi:10.1016/j.eururo.2015.07.041

6. Andrabi Y, Patino M, Das CJ, Eisner B, Sahani DV, Kambadakone A. Advances in CT imaging for urolithiasis. Indian J Urol. 2015;31 (3):185-193. doi:10.4103/0970-1591.156924

7. Teichman JMH. Clinical practice: acute renal colic from ureteral calculus. $N$ Engl J Med. 2004;350(7):684-693. doi:10.1056/ NEJMcp030813

8. Koteshwar P, Kakkar C, Sripathi S, Parakh A, Shrivastav R. Low dose MDCT with tube current modulation: role in detection of urolithiasis and patient effective dose reduction. $J$ Clin Diagn Res. 2016;10(5):TC01-TC05. doi:10.7860/JCDR/2016/16765.7735

9. Park SB, Kim YS, Lee JB, Park HJ. Knowledge-based iterative model reconstruction (IMR) algorithm in ultralow-dose CT for evaluation of urolithiasis: evaluation of radiation dose reduction, image quality, and diagnostic performance. Abdom Imaging. 2015;40 (8):3137-3146. doi:10.1007/s00261-015-0504-y

10. Scott Kriegshauser J, Naidu SG, Paden RG, He M, Wu Q, Hara AK. Feasibility of ultra-low radiation dose reduction for renal stone CT using model-based iterative reconstruction: prospective pilot study. Clin Imaging. 2015;39(1):99-103. doi:10.1016/j.clinimag.2014.10.013

11. Desai GS, Uppot RN, Yu EW, Kambadakone AR, Sahani DV. Impact of iterative reconstruction on image quality and radiation dose in multidetector CT of large body size adults. Eur Radiol. 2012;22 (8):1631-1640. doi:10.1007/s00330-012-2424-3 
12. Soenen O, Balliauw C, Oyen R, Zanca F. Dose and image quality in low-dose ct for urinary stone disease: added value of automatic tube current modulation and iterative reconstruction techniques. Radiat Prot Dosimetry. 2017;174(2):242-249. doi:10.1093/rpd/ncw133

13. Chang DH, Hiss S, Mueller D. Radiation dose reduction in computed tomography-guided lung interventions using an iterative reconstruction technique. Rofo. 2015;187(10):906-914.

14. Gay F, Pavia Y, Pierrat N, Lasalle S, Neuenschwander S, Brisse HJ. Dose reduction with adaptive statistical iterative reconstruction for paediatric CT: phantom study and clinical experience on chest and abdomen CT. Eur Radiol. 2014;24(1):102-111. doi:10.1007/s00330-013-2982-z

15. Deak PD, Smal Y, Kalender WA. Multisection CT protocols: sex- and age-specific conversion factors used to determine effective dose from dose-length product. Radiology. 2010;257(1):158-166. doi:10.1148/ radiol. 10100047

16. Trinchieri A, Coppi F, Montanari E, Del Nero A, Zanetti G, Pisani E. Increase in the prevalence of symptomatic upper urinary tract stones during the last ten years. Eur Urol. 2000;37(1):23-25. doi:10.1159/ 000020094

17. Stamatelou KK, Francis ME, Jones CA, Nyberg LM, Curhan GC. Time trends in reported prevalence of kidney stones in the United States: 1976-1994. Kidney Int. 2013;63(5):1817-1823. doi:10.1046/ j.1523-1755.2003.00917.x

18. Ogden CL, Carroll MD, Curtin LR, McDowell MA, Tabak CJ, Flegal KM. Prevalence of overweight and obesity in the United States, 1999-2004. JAMA. 2006;295(13):1549-1555. doi:10.1001/ jama.295.13.1549

19. Ford ES, Giles WH, Dietz WH. Prevalence of the metabolic syndrome among US adults: findings from the National Health and Nutrition Examination Survey. JAMA. 2002;287(3):356-359.

20. Borysewicz-Sańczyk H, Porowski T, Hryniewicz A, Baran M, Zasim A, Bossowski A. Urolithiasis risk factors in obese and overweight children Pediatr Endocrinol Diabetes Metab. 2012;18(2):53-57.

21. Negri AL, Spivacow FR, Del Valle EE, Forrester M, Rosende G, Pinduli I. Role of overweight and obesity on the urinary excretion of promoters and inhibitors of stone formation in stone formers. Urol Res. 2008;36(6):303-307. doi:10.1007/s00240008-0161-5
22. Elkoushy MA, Andonian S. Lifetime radiation exposure in patients with recurrent nephrolithiasis. Curr Urol Rep. 2017;18(11):85. doi:10.1007/s11934-017-0731-6

23. Tonolini M, Valconi E, Vanzulli A, Bianco R. Radiation overexposure from repeated CT scans in young adults with acute abdominal pain. Emerg Radiol. 2018;25(1):21-27. doi:10.1007/s10140-0171554-y

24. Ferrandino MN, Bagrodia A, Pierre SA, et al. Radiation exposure in the acute and short-term management of urolithiasis at 2 academic centers. J Urol. 2009;181(2):668-672. doi:10.1016/j.juro.2008.10.012

25. Hur J, Park SB, Lee JB, et al. CT for evaluation of urolithiasis: image quality of ultralow-dose (Sub mSv) CT with knowledge-based iterative reconstruction and diagnostic performance of low-dose CT with statistical iterative reconstruction. Abdom Imaging. 2015;40 (7):2432-2440. doi:10.1007/s00261-015-0411-2

26. Kulkarni NM, Uppot RN, Eisner BH, Sahani DV. Radiation dose reduction at multidetector $\mathrm{CT}$ with adaptive statistical iterative reconstruction for evaluation of urolithiasis: how low can we go? Radiology. 2012;265(1):158-166. doi:10.1148/radiol.12112470

27. Son JH, Kim SH, Yoon J-H, Lee Y, Lim Y-J, Kim S-J. Comparison of model-based iterative reconstruction, adaptive statistical iterative reconstruction, and filtered back projection for detecting hepatic metastases on submillisievert low-dose computed tomography. J Comput Assist Tomogr. 2017;41(4):644-650. doi:10.1097/RCT.0000000000000577

28. Botsikas D, Stefanelli S, Boudabbous S, Toso S, Becker CD, Montet X. Model-based iterative reconstruction versus adaptive statistical iterative reconstruction in low-dose abdominal CT for urolithiasis. AJR Am J Roentgenol. 2014;203(2):336-340. doi:10.2214/AJR.13.11937

29. Khawaja RDA, Singh S, Blake M, et al. Ultra-low dose abdominal MDCT: using a knowledge-based iterative model reconstruction technique for substantial dose reduction in a prospective clinical study. Eur J Radiol. 2015;84(1):2-10. doi:10.1016/j. ejrad.2014.09.022

30. Youn JH, Kim SS, Yu JH, Sung LH, Noh CH, Chung JY. Efficacy and safety of emergency ureteroscopic management of ureteral calculi. Korean J Urol. 2012;53(9):632-635. doi:10.4111/kju.2012.53.9.632
Diabetes, Metabolic Syndrome and Obesity: Targets and Therapy is an international, peer-reviewed open-access journal committed to the rapid publication of the latest laboratory and clinical findings in the fields of diabetes, metabolic syndrome and obesity research. Original research, review, case reports, hypothesis formation, expert opinion and commentaries are all considered for publication. The manuscript management system is completely online and includes a very quick and fair peer-review system, which is all easy to use. Visit http://www.dovepress.com/testimonials.php to read real quotes from published authors. 